\title{
Method for obtaining structure and interactions from oriented lipid bilayers
}

\author{
Yulia Lyatskaya, ${ }^{1}$ Yufeng Liu, ${ }^{1}$ Stephanie Tristram-Nagle, ${ }^{2}$ John Katsaras, ${ }^{3}$ and John F. Nagle ${ }^{1,2}$ \\ ${ }^{1}$ Department of Physics, Carnegie Mellon University, Pittsburgh, Pennsylvania 15213 \\ ${ }^{2}$ Department of Biological Sciences, Carnegie Mellon University, Pittsburgh, Pennsylvania 15213 \\ ${ }^{3}$ National Research Council, Steacie Institute for Molecular Sciences, Neutron Program for Materials Research, \\ Chalk River Laboratories Building 459, Station 18, Chalk River, Ontario, Canada KOJ $1 \mathrm{JO}$
}

(Received 13 July 2000; published 22 December 2000)

\begin{abstract}
Precise calculations are made of the scattering intensity $I(\mathbf{q})$ from an oriented stack of lipid bilayers using a realistic model of fluctuations. The quantities of interest include the bilayer bending modulus $K_{c}$, the interbilayer interaction modulus $B$, and bilayer structure through the form factor $F\left(q_{z}\right)$. It is shown how $K_{c}$ and $B$ may be obtained from data at large $q_{z}$ where fluctuations dominate. Good estimates of $F\left(q_{z}\right)$ can be made over wide ranges of $q_{z}$ by using $I(\mathbf{q})$ in $\mathbf{q}$ regions away from the peaks and for $q_{r} \neq 0$ where details of the scattering domains play little role. Rough estimates of domain sizes can also be made from smaller $q_{z}$ data. Results are presented for data taken on fully hydrated, oriented DOPC bilayers in the $L_{\alpha}$ phase. These results illustrate the advantages of oriented samples compared to powder samples.
\end{abstract}

DOI: 10.1103/PhysRevE.63.011907 PACS number(s): 87.16.-b, 87.64.Bx, 61.30.-v, 61.10.Dp

\section{INTRODUCTION}

Lipid bilayers have been much studied as the prototypical biomembrane. One focus of such studies is the structure of lipid bilayers and how that structure varies with the thermodynamic phase and with the chemistry of different lipids $[1,2]$. Another focus is on the interactions between two bilayers $[3,2]$ and on the mechanical properties of bilayers [4].

For these purposes many groups have used x-ray scattering from lipid bilayers in the model system of multilamellar vesicles (MLVs) [1-3,5]. Each MLV consists of bilayers arranged (topologically at least) as more than 200 (usually many more) concentric spheres with an average repeat spacing $D$ of order $60 \AA$, of which about $25 \AA$ is water, depending upon the lipid and the phase. For scattering purposes MLV samples effectively consist of independent domains, each of which is a stack of parallel bilayers, but the normals to the bilayers in the independent stacks are isotropically distributed in space. Such powder samples diffract relatively weakly and the intensity falls off rapidly with $q$ because of the Lorentz factor which scales as $1 / q^{2}$. For samples in the fully hydrated, most biologically relevant, fluid $\left(L_{\alpha}\right)$ phase, often only $h=2$ orders of diffraction are observable $\left(q_{z, \max }\right.$ $\left.\approx 0.2 \AA^{-1}\right)$. Dehydrating the sample by applying osmotic pressure increases the number of observable orders, but there is an osmotic pressure correction to structure that must be applied $[2,6,7]$. Furthermore, for at least one lipid (DOPC), the structure changes drastically upon mild dehydration (relative humidity near 96\%) [8]; for lower relative humidity, diffraction data are not relevant for the desired bilayer structure [5].

The intensity of diffraction is much greater from stacks of bilayers that are oriented with their normals aligned along one axis. However, until quite recently there has been concern about studying oriented samples by $\mathrm{x}$-ray diffraction because they could not be hydrated from water vapor to the full $D$ spacing of samples immersed in water. This "vapor pressure paradox" has recently been shown to be an experimental artifact $[9,10]$ and sample chambers suitable for x-ray studies have now been built that obtain full hydration [11]. Figure 1 shows the kind of data that hold the promise of providing far more information than the MLV samples. The purpose of this paper is to show how data like those in Fig. 1 can be analyzed.

The first reason that the data in Fig. 1 are so promising is that there is intensity for a $q z$ range at least up to $0.6 \AA^{-1}$, more than twice as far as for the comparable MLV samples [12]. The second reason is that the deep minima in the intensities near $q_{z}=0.28$ and $0.44 \AA^{-1}$ immediately show where there are zeros in the single bilayer form factor $F\left(q_{z}\right)$, defined by

$$
F\left(q_{z}\right)=\int_{-\infty}^{\infty}\left[\rho_{I}^{*}(z)-\rho_{W}^{*}\right] \cos \left(z q_{z}\right) d z
$$

$F\left(q_{z}\right)$ provides information about the structure of a bilayer centered at $z=0$ with average normal direction $z$ through the contrast in the electron density $\rho_{I}^{*}(z)$ relative to that of water $\rho_{W}^{*}$. Third, in addition to data in the $q_{z}$ direction, there is clearly distinguishable information in Fig. 1 along the $q_{r}$ direction which, for MLV samples, is lost by convolution into a powder-averaged $q$ dependence [13]. Finally, the data are not confined just to the vicinity of the peaks as in our previous studies on MLVs [13], although other groups [1416] have obtained and analyzed intensity data that are continuous in $q$ for MLV samples.

This work is closest in many ways to the study of Lei et al. [17] and has benefited from it (see especially Lei [18]). There are, however, substantial differences which require a separate development. Lei et al. studied a surfactant system where the bending modulus could be made small by adding cosurfactant so that their inverted bilayers could swell to large $D$ values. This took the system into the hard confinement regime where the Helfrich theory of interactions [19] is appropriate, whereas our systems have much smaller $D$ and are in the soft confinement regime [20,21]. Because fluctuations were large, at most two peaks were observed and data 


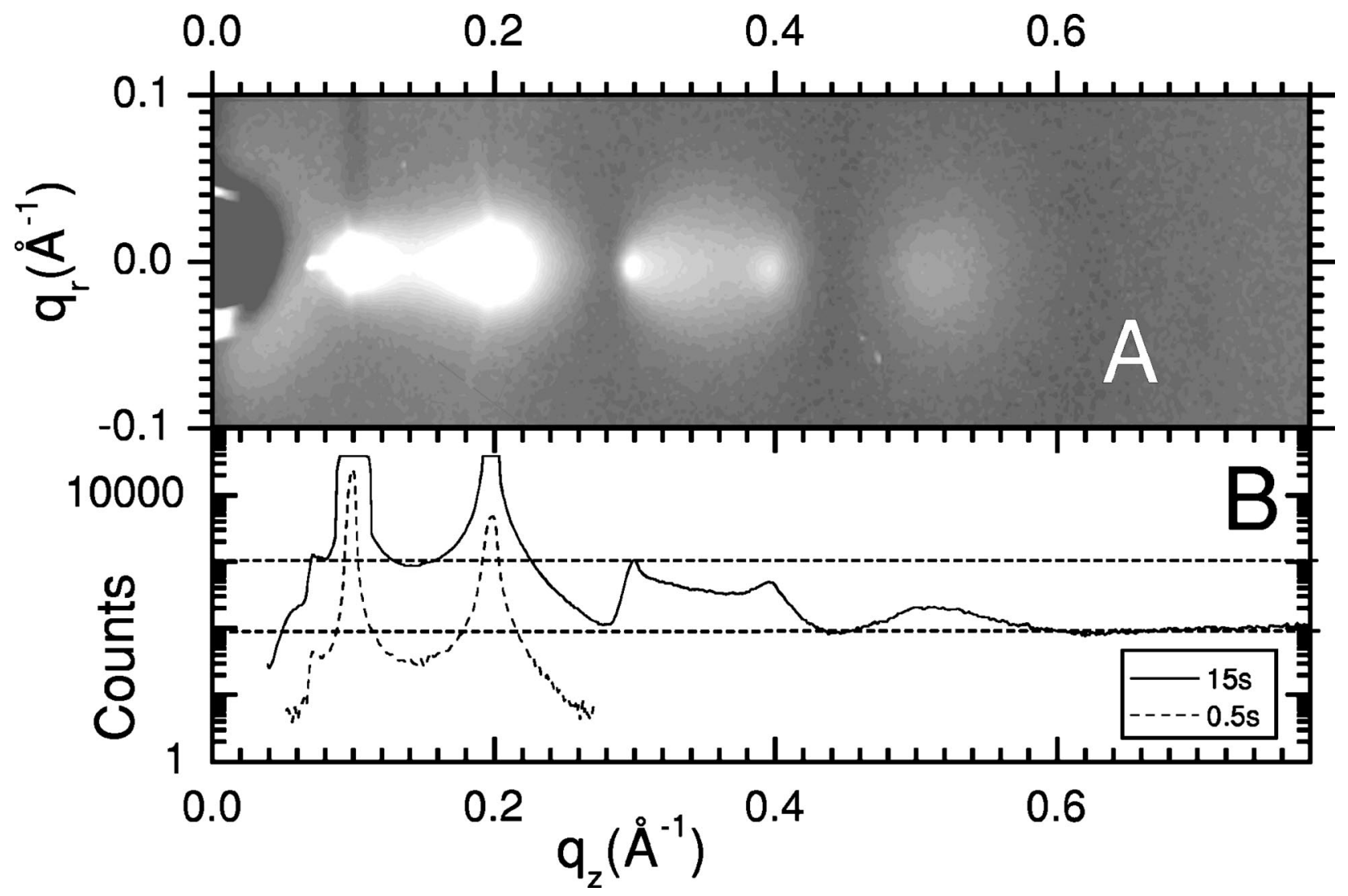

FIG. 1. Scattering intensity from a sample oriented on a cylindrical substrate versus scattering vector, where $q_{z}$ is normal to the stack of bilayers and $q_{r}$ is in the plane of the bilayers. (A) shows high intensities in white and (B) shows the average intensity as a function of $q_{z}$ for a strip of width $\delta q_{r}=0.01 \AA^{-1}$ centered at $q_{r}=0$. The dashed lines in (B) indicate the range for the grey scale in (A). Data are from oriented fully hydrated $L_{\alpha}$ phase DOPC at $30^{\circ} \mathrm{C}$ obtained at CHESS with a CCD detector [30].

extended only to about $0.25 \AA^{-1}$. Structure was not a goal, so the electron density of the bilayer $\rho_{I}^{*}(z)$ was assumed to be a constant which is an adequate assumption for their $q_{z}$ range, but not for ours for which the extended $q$ range provides more information about the structurally useful modulation of $\rho_{I}^{*}(z)$ across the bilayer. Their major goal, which is also one of ours, was to obtain the bending modulus $K_{c}$ and the bulk (interaction) modulus $B$. These two moduli are related to two parameters that were obtained from the scattering data. The first of these is the Caille parameter [22]

$$
\eta_{1}=\frac{k_{B} T}{8 \sqrt{B K_{c}}} \frac{4 \pi}{D^{2}} .
$$

The second, which involves $B$ and $K_{c}$ in a different combination, is an in-plane correlation length $[23,17]$

$$
\xi^{4}=K_{c} / B
$$

which is related to the de Gennes penetration length $\lambda$ [24] by $\xi^{2}=\lambda D$. The Caillé parameter has been obtained in many studies, including MLVs, by analyzing the tails of the diffraction peaks $[13,25]$. Obtaining $\xi$ is even more challenging [26]; in particular, powder data are very insensitive to $\xi$ [13]. Lei et al. [17] obtained $\xi$ by analyzing the intensity in the very small angle regime $\left(q_{z}<0.03 \AA^{-1}\right)$. However, as they noted, this small-angle scattering becomes weak in the more weakly fluctuating soft confinement regime appropriate to our lipid bilayers. We therefore did not attempt to obtain data at such small angles (this small-angle regime is obscured by the beam block in the data in Fig. 1). Instead, we show how both $\eta_{1}$ and $\xi$ (equivalently, $B$ and $K_{c}$ ) can be obtained from the $q_{r}$ dependence of the data at large values of $q_{z}$.

Although the data in Fig. 1 contain a great deal of information, including the structural information in $F\left(q_{z}\right)$ and the interaction information in $\eta_{1}$ and $\xi$, the data are also affected by the domain size. Following [13], we define $L_{z}$ to be the average domain size in the direction of the average bilayer normals. For cylindrical domain shapes, $L_{r}$ is defined to be the average radius in the transverse direction along the plane of the bilayers. We also consider distributions in these two sizes. The data also depend upon inevitable experimental artifacts such as X-ray beam size, mosaic spread, and instrumental resolution. We will initially ignore the experimental issues, except to note that the instrumental resolution for the data in Fig. 1 was limited by the pixel size of the chargecoupled device (CCD) detector, about $0.001 \AA^{-1}$, which is 10 times coarser than our best resolution for MLV samples [12]. Therefore, our analysis must not rely upon the most intense data very near sharp diffraction peaks, but must instead focus upon the weaker intensity outside the peak regions. 
Since the data are affected by so many parameters, the analysis should ultimately consist of a simultaneous fit of a model to the global data. However, in this paper we prefer to establish the feasibility that such a program even has a chance to succeed by identifying different regions of the data from which a smaller subset of parameters can be estimated separately. That this piecemeal approach might work is indicated by the fact that the high- $q_{z}$ data are so strongly affected by fluctuations that all the scattering is diffuse whereas the smaller- $q_{z}$ data exhibit sharper and narrower peaks, more typical of ordered samples. Another indication is that the intensity $I(\mathbf{q})$ is a product

$$
I(\mathbf{q})=S(\mathbf{q})\left|F\left(q_{z}\right)\right|^{2} / q_{z}
$$

of a structure factor $S(\mathbf{q})$, which contains the $B$ and $K_{c}$ information, and a form factor squared, which does not have any $q_{r}$ dependence, as well as the customary $q_{z}^{-1}$ Lorentz factor for oriented samples. This implies that the $q_{r}$ dependence must therefore come from $S(\mathbf{q})$. Therefore, the determination of the structural parameters in $F$ can be separated from the remaining parameters involved in $S$.

In Sec. II the basic model of discrete bilayer stacks is reviewed and it is shown how the correlation functions that affect $\mathrm{x}$-ray scattering are calculated. It is shown how the structure factor $S(\mathbf{q})$ is calculated in Sec. III. Regions in which the domain sizes and distribution have little effect on $S(\mathbf{q})$ are identified in Sec. IV. It is then shown in Sec. V that both $\eta_{1}$ and $\xi$ can be determined from data in these regions. We return in Sec. VI to the other regions where the domain sizes do affect $S(\mathbf{q})$ and show how the sizes can be estimated from data in these regions. It is then shown in Sec. VII how the previous sections enable determination of the form factor $F\left(q_{z}\right)$. Finally, in Sec. VIII this new analysis is used to provide results for the data in Fig. 1.

\section{CORRELATION FUNCTIONS FOR FLUCTUATIONS}

The system consists of $n=1, \ldots, N$ bilayers stacked in the $z$ direction. The center of each bilayer has an average position $z_{n}=n D$ and each bilayer extends over a diameter $L_{r}$ in the $\mathbf{r}=(x, y)$ plane. Fluctuations in the stacking of the bilayers are described by the quantities $u_{n}(\mathbf{r})$ which are spatial deviations in the $z$ direction of the center of the $n$th bilayer from its average position at location $\mathbf{r}$. The original Caillé theory [22] employed a continuum model of smectic liquid crystals which has gradually been replaced by the following, more realistic, discrete free energy functional $[27,20,17,28,21]$

$f=\frac{\pi}{N L_{r}^{2}} \int r d r \sum_{n=0}^{N-1}\left\{K_{c}\left[\nabla_{r}^{2} u_{n}(r)\right]^{2}+B\left[u_{n+1}(r)-u_{n}(r)\right]^{2}\right\}$,

where the first term accounts for the bending energy of individual bilayers and the second term is a harmonic approximation to the interactions between adjacent bilayers [20,28], parametrized by the modulus $B$ which has units of energy/ length ${ }^{4}$. Our $B$ is related to the Caille three-dimensional bulk modulus $B_{3}$ by $B=B_{3} / D$.

To treat $\mathrm{x}$-ray scattering it is necessary to calculate the height-height pair correlation function

$$
\delta u_{n}(r)=\left\langle\left[u_{n}(r)-u_{0}(0)\right]^{2}\right\rangle,
$$

which has no azimuthal dependence because the bilayers are in-plane fluids. We will avoid the ambiguity that arises from imposing boundary conditions on a finite-size domain by calculating the correlation functions for an infinite domain and assume translational invariance. The restriction to finite-size domains will be made in Sec. III by summing the infinite domain correlation functions only over pairs that are contained in such a domain. Then, for the discrete model in Eq. (5) it has been shown $[17,28]$ that

$$
\begin{aligned}
\delta u_{n}(r)= & \frac{4 k_{B} T}{(2 \pi)^{2} B D^{2}} \int_{0}^{\pi / D} d k_{z} \\
& \times \int_{0}^{\pi / a} \frac{k_{r} d k_{r}\left[1-J_{0}\left(k_{r} r\right) \cos \left(k_{z} n D\right)\right]}{4 \sin ^{2}\left(k_{z} D / 2\right)+\xi^{4} k_{r}^{4}},
\end{aligned}
$$

where $J_{0}$ is the zeroth-order Bessel function. Defining $x$ $=\left(\xi k_{r}\right)^{2} / 2$ and integrating over $k_{z}$ using the calculus of residues [18] yields

$$
\begin{aligned}
\delta u_{n}(r)= & \frac{2 \eta_{1}}{q_{1}^{2}} \int_{0}^{(\pi / a)^{2} \xi^{2} / 2} d x \\
& \times \frac{\left[1-J_{0}\left(r \sqrt{2 x / \xi^{2}}\right)\left(\sqrt{1+x^{2}}-x\right)^{2 n}\right]}{x \sqrt{1+x^{2}}},
\end{aligned}
$$

where $q_{1}=2 \pi / D$ is the position of the first diffraction peak. Equation (8) shows that $\eta_{1}$ is just a scaling factor for $\delta u_{n}(r)$. This is computationally convenient because a table of correlation functions can be calculated once to use with different values of $\eta_{1}$. Something similar occurs with the parameter $\xi$, but this is somewhat less obvious, since $\xi$ appears both inside the integral and in the upper limit in Eq. (8). Fortunately, the upper limit of integration can be extended to $\infty$ with high accuracy [18], as we have verified. To remove $\xi$ from inside the integral, we change variables to $t$ $=r / \xi$ so that the height-height correlation functions finally can be written

$$
\delta u_{n}(\xi t)=\frac{2 \eta_{1}}{q_{1}^{2}} \int_{0}^{\infty} d x \frac{\left[1-J_{0}(t \sqrt{2 x})\left(\sqrt{1+x^{2}}-x\right)^{2 n}\right]}{x \sqrt{1+x^{2}}},
$$

in which $\xi$ appears only as a scaling factor of $r$. This means that we can calculate a table for $\delta u_{n}(r)$ only once for certain values of $r=\xi t, n, \eta_{1}$, and $\xi$. Then, we can use this table for other values of $\eta_{1}$ by simply rescaling the values of $\delta u_{n}(r)$ by a constant factor. And we can use this table of $\delta u_{n}(r)$ for other values of $\xi$ by rescaling the $r$ dimension. We calculate $\delta u_{n}(r)$ in Eq. (9) numerically as a function of $r$ and $n$ on a 


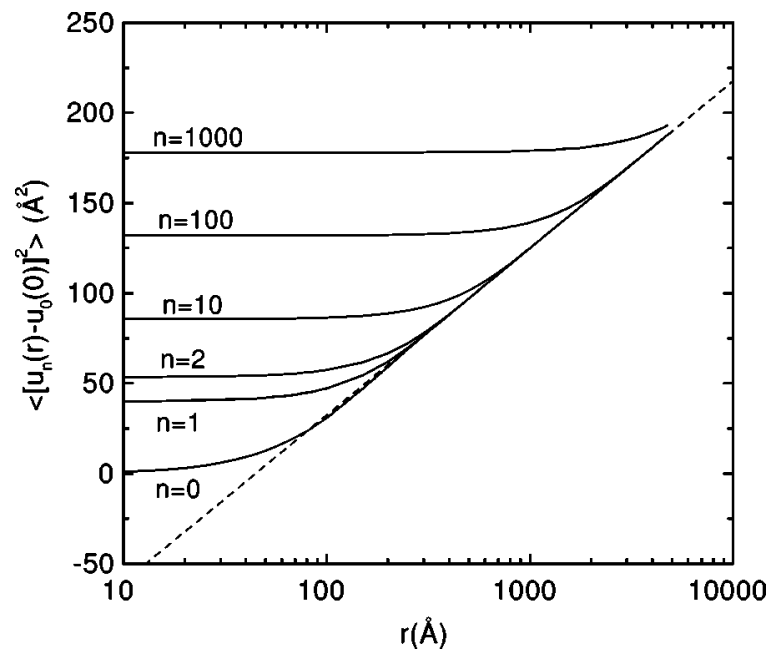

FIG. 2. Correlation functions $\delta u_{n}(r)$ for various values of $n$ (solid lines). The dashed line shows the approximation in Eq. (10) for $n=0$.

grid and save the values in a table. (It is computationally useful to use logarithmic steps in $r$.) We then perform smooth polynomial interpolations for $\delta u_{n}(r)$ for any $r$ between the grid points.

Caillé [22] proposed an approximate correlation function for $r>\xi$,

$$
\delta u_{n}(r)=\frac{4 \eta_{1}}{q_{1}^{2}}\left[\gamma+\ln \left(\frac{r}{\xi}\right)+0.5 E_{1}\left(\frac{r^{2}}{4 n \xi^{2}}\right)\right],
$$

where $\gamma$ is Euler's constant and $E_{1}(t)$ is the exponential integral. In Fig. 2 we compare this approximation to our accurate results for $n=0$. Even for this worst case good agreement is obtained for $r>1000 \AA$. For $n>0$ the approximation works well for even smaller values of $r$. However, precise numerical calculation is required in the vicinity of $r$ $=0$ and $n=0$ and also high accuracy is needed for detailed analysis of the structure factor, so we use Eq. (9) to calculate $\delta u_{n}(r)$ for $r<2000 \AA$ and $n<30$. We therefore use the Caillé approximation, Eq. (10), for $r>2000 \AA$ or $n>30$, having checked that the fractional difference is less than $10^{-4}$.

\section{CALCULATION OF THE STRUCTURE FACTOR}

The electron density $\rho_{n}^{*}$ of the $n$th bilayer will be written

$$
\rho_{n}^{*}(z, r)=\rho_{I}^{*}\left[z-n D-u_{n}(r)\right],
$$

where $\rho_{I}^{*}(z)$ is the electron density profile of the bilayer centered at $z=0$. As has been discussed before [13,29], there is little reason to suppose that the local fluctuations in the bilayer shape $\delta \rho_{I}^{*}$ are correlated with the long-wavelength fluctuations $u_{n}(r)$, at least when the bilayers are fully hydrated [5]. However, there is a geometric correction to the projected thickness of undulating bilayers along the average bilayer normal which is correlated with $\left|\nabla u_{n}(r)\right|$ and, when under osmotic pressure, the local bilayer thickness is anticor- related with $\left|\nabla u_{n}(r)\right|[5]$. Both effects are limited to about $2 \%$ for typical lipid bilayers and will be ignored. Then, the basic scattering formula

$$
I(\mathbf{q})=\left\langle\left|\int_{V} \rho(\mathbf{R}) e^{i \mathbf{q} \cdot \mathbf{R}} d^{3} \mathbf{R}\right|^{2}\right\rangle
$$

can be written as Eq. (4) $[13,17]$ where $F\left(q_{z}\right)$ is given by Eq. (1) and the structure factor $S_{L}\left(q_{z}, q_{r}\right)$ is

$$
\begin{aligned}
S_{L}\left(q_{z}, q_{r}\right)= & \sum_{n, m=1}^{N} e^{i q_{z}(n-m) D} \\
& \times \int_{|r|,\left|r^{\prime}\right| \leqslant L_{r} / 2} d^{2} \mathbf{r} d^{2} \mathbf{r}^{\prime} e^{i \mathbf{q}_{\mathbf{r}} \cdot\left(\mathbf{r}-\mathbf{r}^{\prime}\right)} \\
& \times G\left(\left|\mathbf{r}-\mathbf{r}^{\prime}\right|, n-m, q_{z}\right),
\end{aligned}
$$

where $G$ is the scattering pair correlation function:

$$
G\left(r, n, q_{z}\right)=\exp \left[-q_{z}^{2} \delta u_{n}(r) / 2\right]
$$

Since the lipid bilayer is organized as a fluid within the plane of each bilayer and there are no local correlations between molecules in adjacent bilayers, each bilayer is an in-plane powder, the azimuthal angle $\theta$ can be integrated out, and the in-plane vector $\mathbf{r}$ can be replaced by the in-plane distance $r$. As already discussed in Sec. II, the correlation functions depend only upon $(n-m)$ and $\left(r-r^{\prime}\right)$, so the structure factor for a domain of size $L_{r}$ and $L_{z}=N D$ becomes

$$
\begin{aligned}
S_{L}\left(q_{z}, q_{r}\right)= & \pi L_{r}^{2} \sum_{n=-N}^{N-1}(N-n) \cos \left(q_{z} n D\right) \\
& \times \int_{0}^{L_{r}} r d r F_{r}\left(r / L_{r}\right) J_{0}\left(q_{r} r\right) G\left(r, n, q_{z}\right),
\end{aligned}
$$

where $F_{r}(x)$, which is a finite-size effect function in the $r$ direction [18], is zero if $x>1$ and for $x \leqslant 1$

$$
F_{r}(x)=\cos ^{-1}(x)-x \sqrt{1-x^{2}}
$$

We next assume that the distributions of domain sizes $L_{r}$ and $L_{z}$ are independent, so that

$$
S\left(q_{z}, q_{r}\right)=\int_{0}^{\infty} d L_{z} P_{z}\left(L_{z}\right) \int_{0}^{\infty} d L_{r} P_{r}\left(L_{r}\right) S\left(q_{z}, q_{r}\right)
$$

where $P_{z}\left(L_{z}\right)$ and $P_{r}\left(L_{r}\right)$ are distribution functions for $L_{z}$ and $L_{r}$. (One might alternatively assume that the $L_{r}$ and $L_{z}$ sizes are correlated in some fashion.) We will use the Gaussian distributions

$$
P_{r}\left(L_{r}\right)=\frac{1}{\sigma_{r}} \exp \left[-\left(L_{r}-\bar{L}_{r}\right)^{2} / 2 \sigma_{r}^{2}\right]
$$




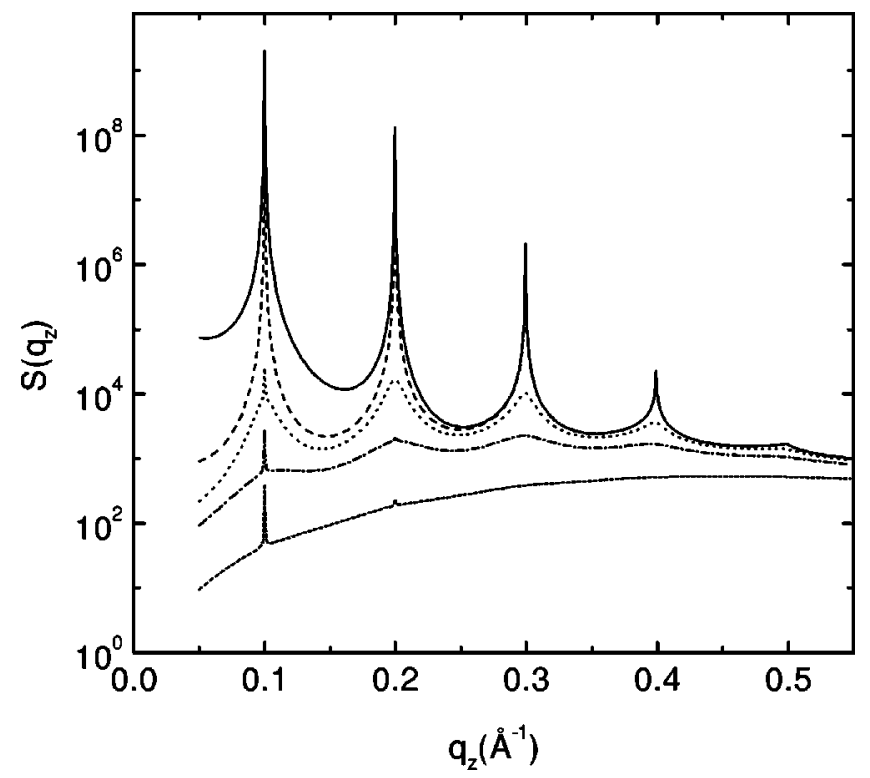

FIG. 3. $S\left(q_{z}\right)$ at various values of $q_{r}\left(\right.$ in $\AA^{-1}$ ) for $D=63 \AA$, $\eta_{1}=0.1, \xi=80 \AA, \bar{L}_{r}=\bar{L}_{z}=10^{4} \AA, \sigma_{z}=\bar{L}_{z} / 3$, and $\sigma_{r}=\bar{L}_{r} / 3$. Solid line: $q_{r}=0$. Dashed line: $q_{r}=0.001 \AA^{-1}$. Dotted line: $q_{r}=0.01$ $\AA^{-1}$. Dash-dotted line: $q_{r}=0.02 \AA^{-1}$. Short dashed line: $q_{r}$ $=0.04 \AA^{-1}$.

$$
P_{z}\left(L_{z}\right)=\frac{1}{\sigma_{z}} \exp \left[-\left(L_{z}-\bar{L}_{z}\right)^{2} / 2 \sigma_{z}^{2}\right]
$$

Equation (17) can be simplified by introducing effective finite-size factors

$$
\begin{gathered}
H_{z}(z)=\int_{z}^{\infty} d L_{z} P_{z}\left(L_{z}\right)\left(L_{z}-z\right) / D, \\
H_{r}(r)=\pi \int_{r}^{\infty} d L_{r} P_{r}\left(L_{r}\right) L_{r}^{2} F_{r}\left(r / L_{r}\right) .
\end{gathered}
$$

Thus, the final equation for $S\left(q_{z}, q_{r}\right)$ becomes

$$
\begin{aligned}
S\left(q_{z}, q_{r}\right)= & \sum_{n=-\infty}^{\infty} H_{z}(n D) \cos \left(q_{z} n D\right) \\
& \times \int_{0}^{\infty} r d r H_{r}(r) J_{0}\left(q_{r} r\right) G\left(r, n D, q_{z}\right) .
\end{aligned}
$$

To calculate $S\left(q_{z}, q_{r}\right)$ from Eq. (22) numerically we first calculate $G$ using the table for $\delta u_{n}(r)$ in Sec. II. We also calculate $H_{z}(z)$ and $H_{r}(r)$ using Eqs. (20) and (21) and save them in tables. We then calculate the integral and sum in Eq. (22) numerically. The accuracy of our calculations has been checked in two ways. The same integration was performed using different tables calculated using a finer grid for $r$ in $\delta u_{n}(r)$ and different integration procedures of the Romberg type have been used, with cutoffs adjusted to provide an accuracy of $10^{-4}$.

Figure 3 shows calculated structure factors where the values of $\eta_{1}$ and $\xi$ were chosen to correspond to $K_{c}=10^{-12}$

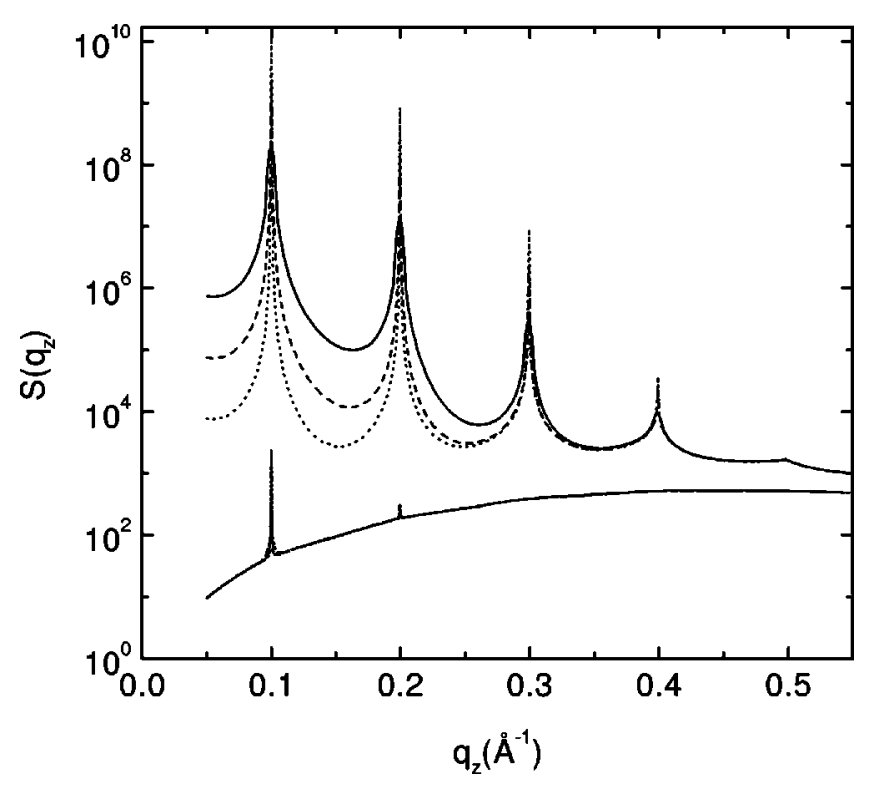

FIG. 4. $S\left(q_{z}\right)$ at different values of $L_{z}$ at $q_{r}=0$ (the upper three curves) and $q_{r}=0.04 \AA^{-1}$ (the lower three curves). Solid line: $L_{z}$ $=10^{3} \AA$, dashed line: $L_{z}=10^{4} \AA$. Dotted line: $L_{z}=10^{5} \AA$.

ergs and $B=2.8 \times 10^{12} \mathrm{ergs} / \mathrm{cm}^{4}$, which are typical for lipid bilayers [28]. As expected [17,13], the intensities of the higher orders rapidly decrease and almost disappear by $h$ $=5$ even at $q_{r}=0$. As $q_{r}$ increases, the higher-order peaks disappear completely, but the first two peaks are seen even at $q_{r}=0.04 \AA^{-1}$. Curiously, the shape of the peaks as a function of $q_{z}$ becomes narrower and more Bragg like as $q_{r}$ increases. In the next several sections we will discuss how changes in the various parameters affect the structure factor shown in Fig. 3.

\section{EFFECT OF DOMAIN SIZES}

Figure 4 shows how the structure factor is affected by the average domain size in the $z$ direction which henceforth will be designated just as $L_{z}$ with no overbar. For all comparisons we normalize $S\left(q_{z}, q_{r}\right)$ by the amount of material in the bilayer stack. This normalization gives the same values for $S\left(q_{z}, q_{r}\right)$ for large values of $q_{z}$. As expected, the peaks also become higher with larger domain size. Because of the overall conservation of scattering intensity, the diffuse scattering between peaks becomes lower with an increase in $L_{z}$. Most importantly, the dependence on $L_{z}$ becomes smaller as $q_{z}$ and/or $q_{r}$ increases. There is almost no dependence between the $h=3$ and $h=4$ peaks when $q_{r}=0$. For $q_{r}>0.01 \AA^{-1}$ there is almost no dependence on $L_{z}$ except very close to $q_{z}=2 \pi h / D$ for $h=1$ and $h=2$.

Figure 5 shows that $L_{r}$ affects the structure factor quite differently from how $L_{z}$ affects it. An increase in $L_{r}$ results in an increase of the whole $S\left(q_{z}\right)$ curve when $q_{r}=0$, more so at low $q_{z}$ values than at high $q_{z}$. This does not violate conservation of total scattering intensity since an increase in $L_{r}$ makes the peaks in $S\left(q_{r}\right)$ sharper and higher, therefore reducing the intensity in the $q_{r}$ direction. Most importantly, and similar to the effect of $L_{z}$ above, the dependence on $L_{r}$ 


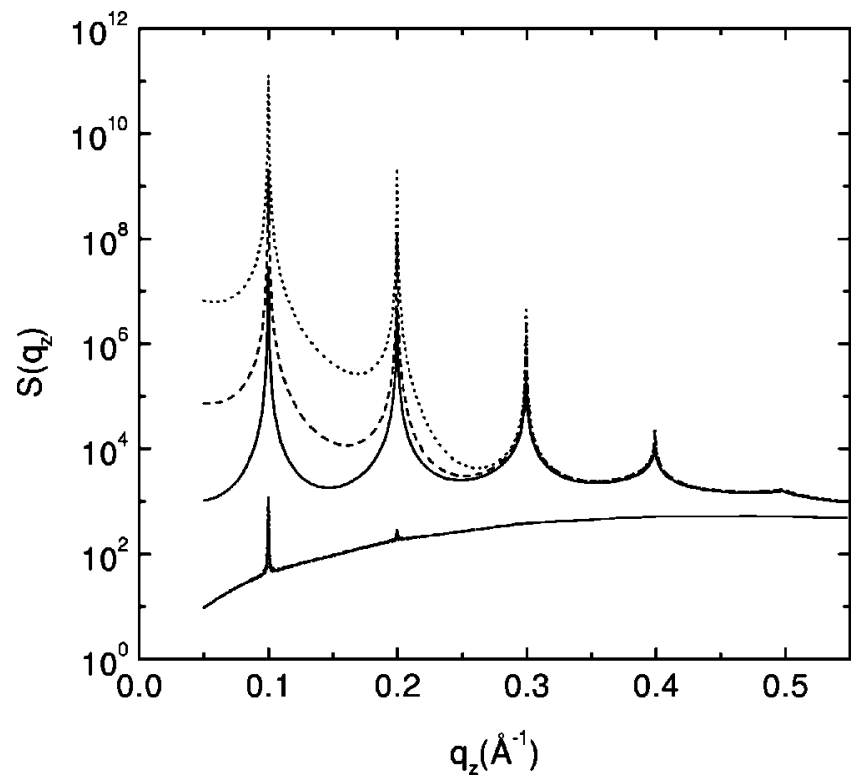

FIG. 5. $S\left(q_{z}\right)$ at different values of $L_{r}$ at $q_{r}=0$ (the upper three curves) and $q_{r}=0.04 \AA^{-1}$ (the lower three curves). Solid line: $L_{r}$ $=5 \times 10^{3} \AA$, Dashed line: $L_{r}=10^{4} \AA$. Dotted line: $L_{r}=6 \times 10^{4} \AA$.

becomes smaller as $q_{z}$ and/or $q_{r}$ increase. There is almost no dependence between the $h=3$ and $h=4$ peaks when $q_{r}$ $=0$. For $q_{r}>0.01 \AA^{-1}$ there is almost no dependence on $L_{r}$ except very close to $q_{z}=2 \pi h / D$ for $h=1$ and $h=2$.

\section{DETERMINATION OF $\eta_{1}$ AND $\xi$}

The results in the previous section show that the domain size parameters can be ignored in large regions of the data where the fluctuations and diffuse scattering dominate. The important unknowns in these regions are therefore $\eta_{1}, \xi$, and the form factor $F\left(q_{z}\right)$. Since $F$ depends only upon $q_{z}$ [Eq. (4)], we now concentrate on the $q_{r}$ dependence of the structure factor to evaluate the possibility of finding $\eta_{1}$ and $\xi$ without having to do a global fit that includes $F\left(q_{z}\right)$ and the domain size parameters.

Figure 6 shows $S$ as a function of $q_{r}$ in regions where the dependence on domain sizes is negligible. Since the overall amplitude of these curves would be set by the as yet unknown form factor, the curves are normalized at $q_{r}=0.01$ $\AA^{-1}$ in order to concentrate on the $q_{r}$ functional dependence for larger $q_{r}$. Figure 6 shows that both parameters $\eta_{1}$ and $\xi$ affect $S\left(q_{r}\right)$. There is an important difference, however, between the effect in Fig. 6(a), which is for a $q_{z}$ slice (constant $q_{z}$ and varying $q_{r}$ ) at the $h=$ fourth-order peak, compared to the effect in Fig. 6(b), which is for a $q_{z}$ slice that goes midway between the third and fourth peaks. In Fig. 6(a) the effect of changing $\eta_{1}$ by a factor of 2 is relatively larger than changing $\xi$ by the same factor and the reverse is true in Fig. 6(b). Therefore, the effect of changing the two parameters is not functionally degenerate and it is therefore plausible that both parameters can be extracted from two $q_{z}$ slices of data.

Programs were written to fit data from two or more $q_{z}$ slices and the programs were tested on calculated data with random noise comparable to the noise in the data in Fig. 1.

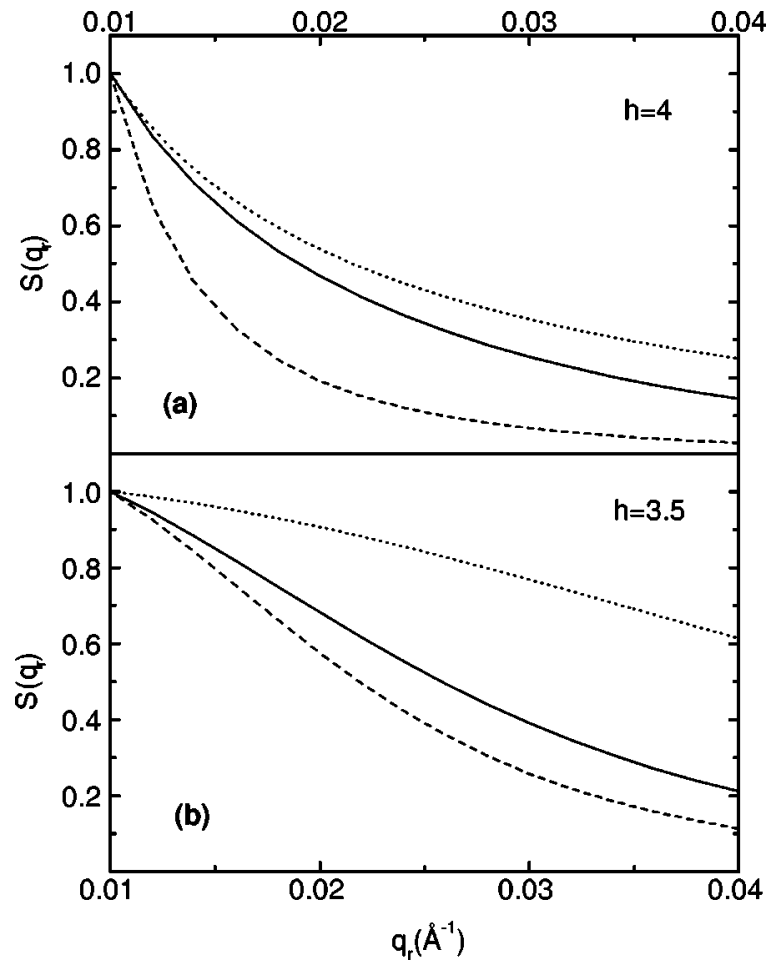

FIG. 6. $S\left(q_{r}\right)$ normalized at $q_{r}=0.01 \AA^{-1}$ for three combinations of $\left(\eta_{1}, \xi\right)$ (i) $(0.1,80 \AA)$ (solid lines), (ii) $(0.1,40 \AA)$ (dotted lines), and (iii) $(0.05,80 \AA)$ (dashed lines). Panel (a) is for the slice $q_{z}=4 q_{1}$ and panel (b) is for the slice $q_{z}=3.5 q_{1}\left(q_{1}=2 \pi / D\right) . L_{z}$ $=10^{4} \AA=L_{r}$.

(We note that data can be taken in future with less noise.) Two slices of calculated data are shown in Fig. 7. The fitted curves are also shown in Fig. 7 and the fitted values of $\eta_{1}$ and $\xi$ agree well with the input values.

We note that a choice of two $q_{z}$ slices should include at least one with $q_{z}$ near a peak value $h q_{1}$ and one from be-

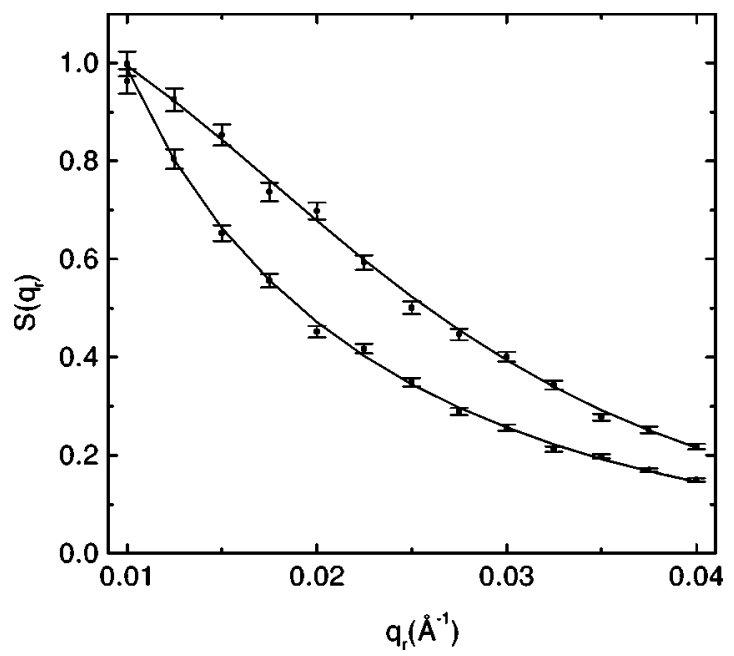

FIG. 7. Calculated data points, normalized at $q_{r}=0.01 \AA^{-1}$, with added noise for $\eta_{1}=0.1$ and $\xi=80 \AA$ are shown by points with error bars for two slices $q_{z}=4 q_{1}$ (lower data) and $q_{z}=3.5 q_{1}$ (upper data). A nonlinear least-squares fit to the data produced the two solid curves and returned values $\eta_{1}=0.096$ and $\xi=78.9 \AA$. 


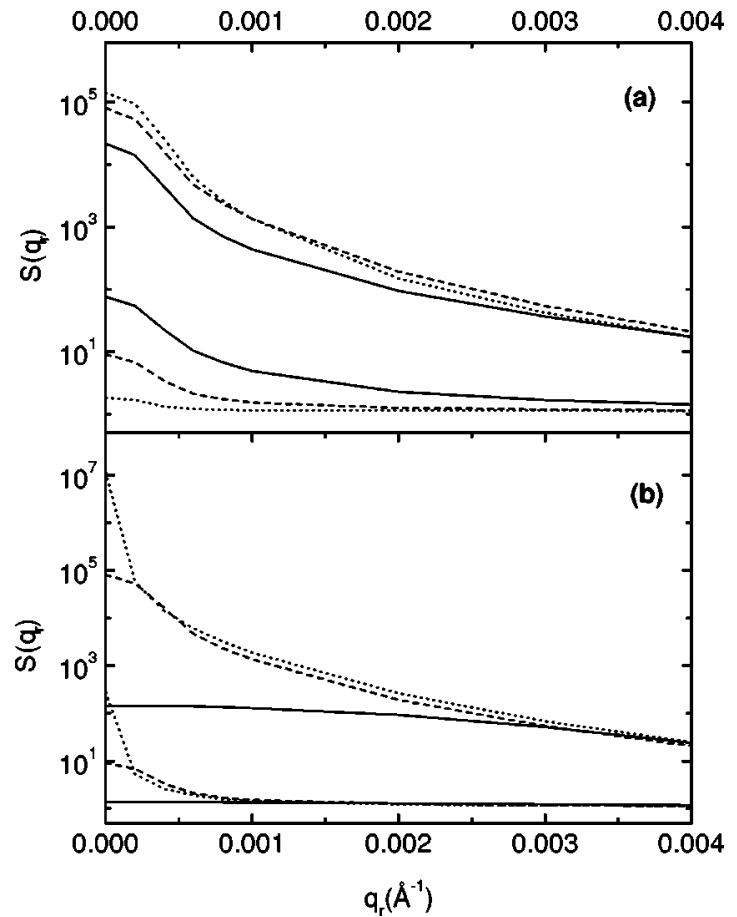

FIG. 8. Structure factor as a function of $q_{r}$ for $q_{z}=2 \pi / D$ (upper curves) and $q_{z}=3 \pi / D$ (lower curves). (a) shows the effect of changing $L_{z}$ with constant $L_{r}=10^{4} \AA$ and (b) shows the effect of changing $L_{r}$ with constant $L_{z}=10^{4} \AA$. Solid lines are for $10^{3} \AA$, dashed lines are for $10^{4} \AA$, and dotted lines are for $10^{5} \AA$. All curves have been normalized to 1 at $q_{r}=0.01 \AA^{-1}$.

tween peaks, such as $\left(h-\frac{1}{2}\right) q_{1}$, as shown in Figs. 6 and 7 . Choosing two slices, both between successive peaks, does not give sufficient contrast to obtain good parameter values when noise is included. We have used more than two $q_{z}$ slices to increase the accuracy of the determination of the parameters, but two slices suffice to illustrate the principle.

\section{DETERMINATION OF DOMAIN SIZES}

In Sec. IV the emphasis on the effect of domain sizes was to find the scattering regions where the domain sizes $L_{z}$ and $L_{r}$ had little effect so that the evaluation of $\eta_{1}$ and $\xi$ could be carried out independently as shown in Sec. V. We now turn to the scattering regions near and between the low-order peaks where the domain size has a large effect. This will give us a method to estimate domain sizes. This procedure uses the values of $\eta_{1}$ and $\xi$ that will now be assumed to be determined by the method of Sec. V. We cannot assume the values of the form factors $F\left(q_{z}\right)$, so the method in this section will again focus on the $q_{r}$ dependence of the structure factor but now at low values of $q_{z}$.

Figure 8(a) shows the dependence of $S\left(q_{r}\right)$ on $L_{z}$ for two $q_{z}$ slices, one that goes through the $h=1$ peak and one that goes halfway between the $h=1$ and $h=2$ peaks. We have chosen to normalize these curves to unity at $q_{r}=0.01 \AA^{-1}$, which is the same value of $q_{r}$ used for normalization in Sec. $\mathrm{V}$, since there is little dependence of $S$ on domain size for $q_{r}>0.01$. With this normalization the dependence of $S$ on $L_{z}$ near $q_{r}=0$ is opposite for the two $q_{z}$ slices. Figure $8(\mathrm{~b})$ shows a much different dependence of $S\left(q_{r}\right)$ on $L_{r}$ for the same normalization. For both $q_{z}$ slices an increase in $L_{r}$ results in an increase of $S$ near $q_{r}=0$.

The aforementioned difference in the effect of domain sizes $L_{z}$ versus $L_{r}$ on the behavior of $S\left(q_{r}=0\right) / S\left(q_{r}\right.$ $=0.01)$ at different $q_{z}$ values indicates that these two parameters are functionally inequivalent and therefore both $L_{r}$ and $L_{z}$ should be extractable from the data, as we have verified for calculated data in which we have varied $L_{z}$ and $L_{r}$. However, this assumes that we know the functional form of domain sizes. Our experience with powder samples has led us from Gaussian distributions [12] to exponential distributions [28]. It is also not clear how $L_{r}$ and $L_{z}$ should be correlated. We think it is unlikely that these kinds of issues will be definitively resolved. Fortunately, these domain details are also the least important from the fundamental point of view, since, due to the exigencies of sample preparation, they are strongly variable even for nominally identical samples, as was earlier emphasized for powder samples [25]. (One would expect oriented samples to have even more variation.) Therefore, we are content to obtain a rough estimate of domain sizes and distributions and to focus on data in the regions where details of the domains are not important.

\section{DETERMINATION OF THE FORM FACTOR}

Since $S(\mathbf{q})$ is determined from the methods in the previous sections, we will now use Eq. (4) to calculate the form factor $F\left(q_{z}\right)$, which is the only remaining unknown. As noted at the end of the previous section, because details of domain sizes and distributions are the most problematical quantities to obtain precisely, it is appropriate to determine $F\left(q_{z}\right)$ by analyzing data in regions in $\mathbf{q}$ where such details are unimportant. We have chosen to use $I(\mathbf{q})$ data for the region $0.035<q_{r}<0.040 \AA^{-1}$ where intensity data are also strong enough to perform reasonable fits. For each value of $q_{z}, F\left(q_{z}\right)$ is chosen to provide the best overall fit to Eq. (4) in this $q_{r}$ range.

We have tested this method of finding $F\left(q_{z}\right)$ using calculated data with added noise. As expected, the method works well when one knows $\eta_{1}, \xi, L_{z}$, and $L_{r}$, precisely. Figure 9 shows the result of a more challenging test which supposes that an incorrect value of $L_{r}$ and $L_{z}$ was obtained from the method in Sec. VI. In this test the correct value of the form factor is $F=1$ for all $q_{z}$. Figure 9(a) shows that the estimated $F\left(q_{z}\right)$ is quite good everywhere except very near the diffraction peaks, even though $L_{z}$ was misset by a factor of 5 . Figure 9(b) indicates that missetting $L_{r}$ has a larger, more systematic effect, but it is still confined to the vicinity of each diffraction peak. We note that the values of $F$ in Fig. 9(b) are consistently a few percent too high for most values of $q_{z}$ not near a diffraction peak, but such a uniform error is unimportant since experimental intensities are relative. Since $F\left(q_{z}\right)$ is a smooth function on the $q_{z}$ scale of each of the deviations in Fig. 9, one can easily interpolate accurate values of $F\left(q_{z}\right)$ for these regions where one expects the method to be inaccurate. 


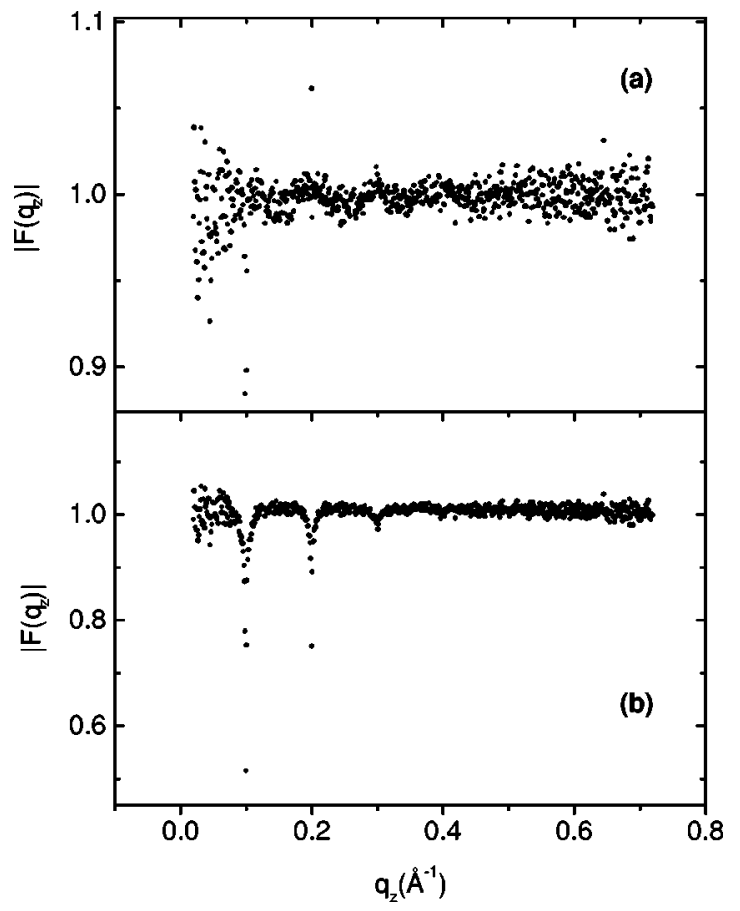

FIG. 9. Form factor $F\left(q_{z}\right)$ obtained by analyzing data calculated with $\eta_{1}=0.1, \xi=80 \AA$, and $L_{r}=L_{z}=10^{4} \AA$ with calculations with (a) $L_{z}$ misset to $2000 \AA$ and (b) $L_{r}$ misset to $2000 \AA$. The true value is $F\left(q_{z}\right)=1$ for all $q_{z}$ and the diffraction peaks occur at $q_{z}=0.1 \mathrm{~h}$ $\AA^{-1}$.

\section{APPLICATION TO DOPC DATA}

We have applied the analysis methods developed above to the data shown in Fig. 1. The instrumental resolution of these data was limited to $\delta q=0.001 \AA^{-1}$ by the pixel size $(50 \mu \mathrm{m}$ of the CCD detector and its placement $(22 \mathrm{~cm})$ from the sample. The sample volume was effectively $10 \mu \mathrm{m}$ $\times 500 \mu \mathrm{m}$ and the latter length was convolved into the calculations to fit the data. The small mosaic spread, estimated to be less than one degree, was not included in the fit.

The results for the fluctuation parameters are $\eta_{1}=0.08$ \pm 0.01 and $\xi=59 \pm 2 \AA$. The value of $\eta_{1}$ may be compared with the value $\eta_{1}=0.10 \pm 0.01$ obtained from the analysis of peak shapes of unoriented fully hydrated MLV samples [6]. Using Eqs. (2) and (3) gives $K_{c}=0.73 \pm 0.04 \times 10^{-12} \mathrm{erg}$ and $B=6.0 \pm 0.7 \times 10^{12} \mathrm{ergs} / \mathrm{cm}^{4}$. This value of $K_{c}$ may be compared with a recent value $K_{c}=0.85 \pm 0.1 \times 10^{-12}$ erg obtained using the aspiration pipette method on giant unilamellar vesicles at the lower temperature of $18^{\circ} \mathrm{C}$ [4]. The value of $B$ has not been previously obtained for fully hydrated DOPC, but the new value generally agrees with the range of values reported for egg lecithin [28].

The results for domain sizes are $L_{z}=0.18 \mu \mathrm{m}$ and $L_{r}$ $=0.5 \mu \mathrm{m}$. The thickness of the sample on the curved glass substrate is at most $10 \mu \mathrm{m}$, so the value of $L_{z}$ appears reasonable. We expected a larger size for $L_{r}$. It is noteworthy, however, that $L_{r}$ is still considerably larger than $\xi$ and $L_{z}$ is considerably larger than the interbilayer water spacing of $0.0025 \mu \mathrm{m}$, so a theory of long-range fluctuations is appropriate for this sample.

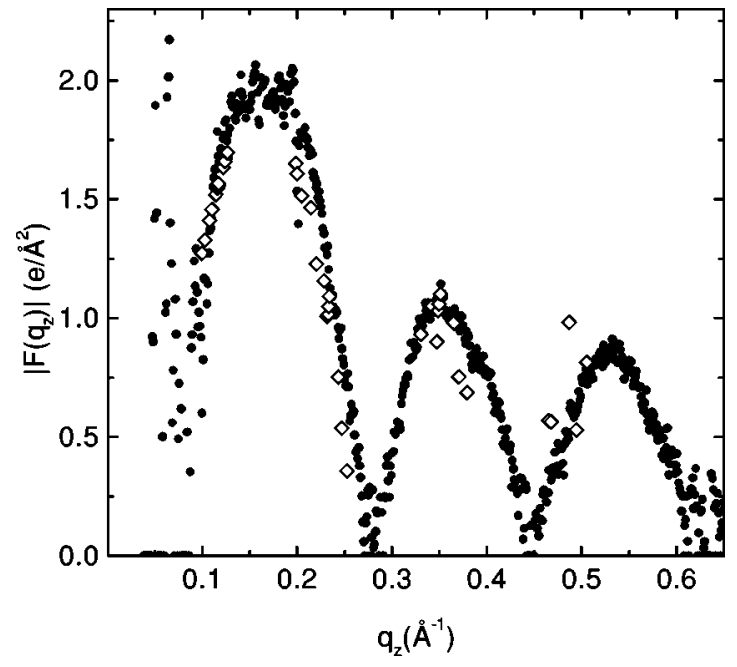

FIG. 10. Absolute value of the form factor $\left|F\left(q_{z}\right)\right|$ for DOPC. Solid circles show results obtained in this work for one fully hydrated sample. Open diamonds show results for many powder samples in different humidity conditions [6].

The result for the form factor is shown in Fig. 10. It is compared to a published form factor obtained for partially dehydrated MLV samples which was put on an absolute scale [6]. The overall scale factor for the new form factor from the oriented sample is set to match the previous form factor in the first lobe region $q_{z}<0.3 \AA^{-1}$ where the MLV results are most accurate. The new results become very noisy for $q_{z}<0.15 \AA^{-1}$ due to uncertainties in subtracting background which is higher for small $q_{z}$ due to air scattering from the beam. The older results in the region of the third lobe $\left(q_{z}>0.44 \AA^{-1}\right)$ were obtained with very few data with large statistical deviations [6]. The new results for $F$ appear to be more accurate in the high- $q_{z}$ region.

\section{CONCLUSIONS}

It has always been attractive to study lipid bilayers stacked in oriented samples because they scatter more strongly. Now that the vapor pressure paradox has been overcome experimentally $[9,10]$, there is added motivation because these samples can be fully hydrated in the biologically relevant fluid phase. However, the intensity is affected by a convolution of many factors. The analysis presented here shows how to extract both the parameters $K_{c}$ and $B$ from $\mathrm{X}$-ray scattering data from such samples in the fluctuating $L_{\alpha}$ phase. These parameters appear in the fluctuational free energy that has previously been shown $[12,28,6]$ to describe powder samples; however, only one of the parameters could be obtained from the powder data. Being able to obtain both parameters will enhance future studies of the basic interactions between lipid bilayers in which the osmotic pressure will be varied systematically to obtain $B$ as a function of the distance between bilayers [3,2,28].

Our analysis shows how to obtain the continuous form factor $F\left(q_{z}\right)$ in a way that is not very sensitive to uncertainties in determining domain size. Previous determinations of the continuous form factor using powder samples relied on 
only a few orders from many samples, each with a different $D$. In contrast, the results in Fig. 10 come from just one sample. The relative wealth of $q$-space data in Fig. 10 raises several possibilities for obtaining electron density profiles for lipid bilayers. In a subsequent paper we will consider some of these possibilities, employing $F\left(q_{z}\right)$ results that we hope will extend to even higher $q_{z}$.

It has also not escaped our attention that this method should be applicable to more complex biomembranes that include mixtures of lipids, sterols, or antibiotic peptides in the biologically relevant, fully hydrated state.

\section{ACKNOWLEDGMENTS}

We are grateful to Dr. Ernie Fontes for getting us started with the CCD detector and the D-line at CHESS, which is supported by NSF Grant No. DMR-9311772. This research was supported by NIH Grant No. GM44976-10 (J.F.N.).
[1] J. F. Nagle and S. Tristram-Nagle, Curr. Opin. Struct. Biol. 10, 474 (2000).

[2] R. P. Rand and V. A. Parsegian, Biochim. Biophys. Acta 988, 351 (1989).

[3] T. J. McIntosh, Curr. Opin. Struct. Biol. 10, 481 (2000).

[4] W. Rawicz, K. C. Olbrich, T. J. McIntosh, D. Needham, and E. A. Evans, Biophys. J. 79, 328 (2000).

[5] J. F. Nagle and S. Tristram-Nagle, Biochim. Biophys. Acta 1469, 159 (2000).

[6] S. Tristram-Nagle, H. I. Petrache, and J. F. Nagle, Biophys. J. 75, 917 (1998).

[7] H. I. Petrache, S. Tristram-Nagle, and J. F. Nagle, Chem. Phys. Lipids 95, 83 (1998).

[8] K. Hristova and S. H. White, Biophys. J. 74, 2419 (1998).

[9] J. Katsaras, Biophys. J. 75, 2157 (1998).

[10] J. F. Nagle and J. Katsaras, Phys. Rev. E 59, 7018 (1999).

[11] J. Katsaras and M. J. Watson, Rev. Sci. Instrum. 71, 1737 (2000).

[12] R. Zhang, S. Tristram-Nagle, W. Sun, R. L. Headrick, T. C. Irving, R. M. Suter, and J. F. Nagle, Biophys. J. 70, 349 (1996).

[13] R. Zhang, R. M. Suter, and J. F. Nagle, Phys. Rev. E 50, 5047 (1994).

[14] F. Nallet, R. Laversanne, and D. Roux, J. Phys. II 3, 487 (1993).

[15] J. Lemmich, K. Mortensen, J. H. Ipsen, T. Honger, R. Bauer, and O. G. Mouritsen, Phys. Rev. E 53, 5169 (1996).

[16] G. Pabst, M. Rappolt, H. Amenitsch, and P. Laggner, Phys. Rev. E 62, 4000 (2000).

[17] N. Lei, C. R. Safinya, and R. F. Bruinsma, J. Phys. II 5, 1155 (1995).

[18] N. Lei, Ph.D. thesis, Rutgers University, 1993.

[19] W. Helfrich, Z. Naturforsch. A 33, 305 (1978).

[20] R. Podgornik and V. A. Parsegian, Langmuir 8, 577 (1992).

[21] N. Gouliaev and J. F. Nagle, Phys. Rev. Lett. 81, 2610 (1998).

[22] A. Caillé, C. R. Seances Acad. Sci., Ser. B 274, 891 (1972).

[23] R. Lipowsky, in Handbook of Biological Physics, edited by R. Lipowsky and E. Sackmann (Elsevier, New York, 1995), Vol. I, Chap. 11.

[24] P. G. deGennes and J. Prost, The Physics of Liquid Crystals (Oxford University Press, New York, 1993).

[25] J. F. Nagle, R. Zhang, S. Tristram-Nagle, W. Sun, H. I. Petrache, and R. M. Suter, Biophys. J. 70, 1419 (1996).

[26] D. C. Wack and W. W. Webb, Phys. Rev. A 40, 1627 (1989).

[27] R. Holyst, Phys. Rev. A 44, 3692 (1991).

[28] H. I. Petrache, N. Gouliaev, S. Tristram-Nagle, R. Zhang, R. M. Suter, and J. F. Nagle, Phys. Rev. E 57, 7014 (1998).

[29] J. F. Nagle, H. I. Petrache, N. Gouliaev, S. Tristram-Nagle, Y. Liu, R. M. Suter, and K. Gawrisch, Phys. Rev. E 58, 7769 (1998).

[30] M. W. Tate, E. F. Eikenberry, S. O. Barna, M. E. Wall, J. L. Lowrance, and S. M. Gruner, J. Appl. Crystallogr. 28, 196 (1995). 